\title{
Enseignement
}

\section{Comment se forme la grêle ? Une activité avec de la pâte à modeler}

\author{
Camille Risi ${ }^{1}$, Frank Roux ${ }^{2}$
}

1. Laboratoire de météorologie dynamique, Institut Pierre-Simon-Laplace, CNRS, Sorbonne

Université, Campus Pierre-et-Marie-Curie, Paris

2. Laboratoire d’aérologie, Université Paul Sabatier Toulouse 3, CNRS, Toulouse

camille.risi@lmd.jussieu.fr

But pédagogique : comprendre comment se forme la grêle

Public : écoles maternelle et primaire

Matériel nécessaire : Pour un groupe de 10 enfants :200 g de pâte à modeler blanche, $200 \mathrm{~g}$ de pâte à modeler bleue, $50 \mathrm{~g}$ de pâte à modeler jaune, 10 petites coupelles, 10 supports imprimés représentant un orage (exemple téléchargeable sur le site web) imprimés si possible sur papier cartonné et plastifié, des tables, une chaise pour chaque enfant, quelques photos d'illustrations (téléchargeables sur le site web), un bac avec de l'eau et des glaçons (facultatif).

Durée de l'activité : 30 minutes à 1 heure.

Site internet : http://www.lmd.jussieu.fr/ crlmd/diff/grelons/grelon.html

Le but de cet article est de présenter un atelier de pâte à modeler visant à comprendre comme se forme la grêle. Les enfants manipulent eux-mêmes la pâte à modeler pour fabriquer chacun un grêlon, et peuvent ensuite repartir avec et le montrer à leurs parents. Cet atelier a l'avantage de pouvoir être proposé à des enfants dès l'école maternelle. Il est inspiré d'une expérience d'illustration présentée dans l'émission E=M6 en mars 2019, et a été testée dans une école maternelle pour des enfants de 3 à 6 ans.

\section{Comment se forme la grêle?}

L'atelier commence par une discussion sur la grêle, illustrée par des photos. Cette section présente un exemple d'enchainement de questions autour desquelles la discussion s'articule, avec leurs réponses. Certaines explications données ici sont trop complexes pour les enfants les plus jeunes, mais nous donnons malgré tout des explications complètes pour que l'enseignant puisse adapter son discours selon le niveau des élèves et qu'il puisse répondre à d’éventuelles questions.

\section{- Avez-vous déjà vu de la grêle?}

Dans l'idéal, cet atelier se déroule au printemps, le lendemain d'une averse de grêle, pour que les enfants aient le souvenir bien frais de ce qu'est de la grêle. Une photo peut aider à leur rafraichir la mémoire (figure 1a). On peut avoir au préalable ramassé un grêlon et l'avoir conservé au congélateur, pour le montrer aux enfants.

On parle de grêle quand les particules glacées ont un diamètre supérieur à $1 \mathrm{~cm}$ (encadré 1$)$. Les gros grêlons peuvent mesurer plusieurs centimètres de diamètre (figure 1b).

- En quelle matière est fait le grêlon?

Il s'agit d'eau glacée. Si l'on montre un vrai grêlon, les enfants pourront vérifier que c'est froid. On peut rappeler les différents états de l'eau et les différents changements de phase. L'eau liquide peut facilement être illustrée avec un verre d'eau, l'eau solide avec des glaçons. Pour la vapeur 
d'eau, invisible dans l'air, on peut s'appuyer sur des questions du type : «où va l'eau du linge mouillé quand il sèche? ».

- Dans quel contexte la grêle se forme-t-elle?

Elle s'observe lorsqu'il y a un orage. Remémorons-nous les éléments d'un orage : un air chaud et humide (le « temps lourd » qu’ont pu évoquer leurs parents), des nuages noirs donc épais, du vent, de la pluie, des éclairs et du tonnerre.

\section{- Comment fonctionne un orage?}

Les orages s'accompagnent de nuages appelés cumulonimbus (figure 1a). Ils ressemblent à des champignons, avec un " pied » surmonté d'un « chapeau » qu'on appelle enclume (figure 1b). La vapeur d'eau est invisible, ce que l'on voit dans le nuage sont les gouttelettes d'eau ou de cristaux de glace en suspension. Dans le « pied » du nuage, l'air monte. La température de l'air diminue et la vapeur d'eau présente dans l'air se condense en eau liquide. Dans les premiers kilomètres de l'atmosphère, la température est supérieure à $0^{\circ} \mathrm{C}$ et il se forme des gouttelettes d'eau. Plus haut dans l'atmosphère, la température est inférieure à $0^{\circ} \mathrm{C}$, et il se forme des cristaux de glace. Au niveau de l'enclume, l'air s'étale latéralement.

Pour expliquer toutes ces notions de manière pédagogique, on peut greffer sur cet atelier plusieurs autres expériences : le nuage dans la bouteille (Risi et al 2014), l'évaporation et la condensation (Risi et al 2016) et le phénomène de convection (http://www.lmd.jussieu.fr/ jldufres/Manip/convect.html).

- Que deviennent les gouttelettes d'eau et les cristaux de glace du nuage d'orage?

Les plus petites gouttelettes d'eau restent en suspension, les plus grosses tombent, formant la pluie. Lorsque les cristaux de glace tombent, ils forment de la neige. Constituée de fin cristaux enchevêtres, cette neige est fragile (encadré 1). Dans les orages de printemps ou d'été, la température ambiante près de la surface est suffisamment chaude pour cette neige fonde avant d'arriver au sol.

\section{- Pourquoi les grêlons ne fondent-ils pas avant d'arriver au sol ?}

Les grêlons n'ont pas le temps de fondre totalement avant d'arriver au sol, parce qu'ils sont plus gros (diamètre supérieur à $1 \mathrm{~cm}$ ) et plus compacts que la neige. Plus les grêlons sont gros, plus ils sont lourds et tombent vite (encadré 2). Donc ils ne fondent pas car ils sont gros, et ils fondent encore moins car ils vont vite.

\section{- Comment les grêlons peuvent-ils devenir aussi gros?}

Pour devenir aussi gros, les grêlons grossissent pendant plusieurs dizaines de minutes. Pendant tout le temps de leur croissance, ils doivent rester en suspension dans le nuage. Vu leur taille et leur poids, il faut qu'ils soient maintenus en suspension dans des courants d'air ascendants très intenses. Par exemple, un grêlon de $4 \mathrm{~cm}$ de diamètre (comme une balle de ping-pong) ne peut se maintenir en suspension que si l'ascendance atteint environ $120 \mathrm{~km} / \mathrm{h}$ (comme une voiture sur l'autoroute) ! (encadré 2).

Des courants d'air ascendants suffisamment rapides et durables n'existent pas dans les cumulonimbus ordinaires (figure 2a). On les rencontre dans des orages très particuliers, qualifiés de supercellulaires ou multi-cellulaires (Roux, 1991). Pour simplifier, nous nous concentrons ici sur le cas des orages super-cellulaires (figure 2c, encadré 3). Ce sont de gros orages très puissants qui tournent sur eux-même, comme une toupie. Au coeur d'un tel orage, on trouve un courant ascendant puissant et durable (figure 2d). Ce sont de ces nuages que tombe la grêle, formant une traînée blanche caractéristique (figure 1c). 
Pour que la glace se forme, le grêlon doit être à une altitude où la température est inférieure à $0^{\circ} \mathrm{C}$ (au dessus de la ligne bleue sur la figure 2d). Pour grandir rapidement, il doit rencontrer d'autres cristaux ou gouttelettes et les « coller » autour de lui. S'il rencontrait d'autres cristaux de glace, ceux-ci rebondiraient lors du choc et ne se colleraient pas. C'est donc en rencontrant des gouttelettes d'eau liquide que le grêlon grossit.

- Comment expliquer la présence d'eau liquide à des températures inférieures à $0^{\circ} \mathrm{C}$ ?

Les petites gouttelettes d'eau présentes dans le nuage congèlent difficilement. Ainsi, il est possible d'observer des gouttes d'eau liquide, dites « surfondues » à des températures inférieures à $0^{\circ} \mathrm{C}$ (encadré 4), souvent entre $0^{\circ}$ et $-25^{\circ} \mathrm{C}$ (entre les lignes bleues et jaunes sur la figure $2 \mathrm{~d}$ ). Si elles rencontrent le grêlon, elles se collent à sa surface et congèlent. C’est ainsi que le grêlon grossit.

- Comment le grêlon en suspension dans le courant ascendant grossit-il ?

Intéressons nous à ce qu'il à l'intérieur d'un grêlon. Si l'on possède un vrai grêlon, on peut le découper en deux. A défaut, on utilise une photo. L'intérieur du grêlon est constitué de couches concentriques, comme des pelures d'oignons (figure 1d). Plus précisément, on observe une alternance de couches transparentes et de couches blanches plus opaques. Ces différentes couches témoignent des différentes conditions que rencontre le grêlon au cours de sa croissance. La couleur de la glace dépend de la quantité de microscopiques bulles d'air qu'elle contient. Une glace transparente contient peu de bulles d'air, tandis qu'une glace opaque blanche en contient beaucoup.

Quand le grêlon en formation traverse une zone concentrée en gouttelettes d'eau surfondues, le grêlon se réchauffe (encadré 4), les gouttelettes gèlent lentement et les bulles d'air s'échappent facilement. La couche de glace ainsi formée est transparente. En revanche, quand le grêlon en formation traverse une zone moins concentrée en gouttelettes, le grêlon se refroidit, les gouttelettes gèlent rapidement et les bulles d'air restent prisonnières. La couche de glace ainsi formée est blanche.

Figure 1. (a) Photo de grêlons. (b) Photo de gros grêlons. ( c) Photo d'un nuage d'orage donnant de la grêle, avec la trainée blanche matérialisant la grêle qui tombe. (d) Coupe transversale d'un grêlon, montrant les couches concentriques.

Figure 2. (a) Photo d'un cumulonimbus isolé. (b) Visualisation du courant ascendant et de l'enclume du cumulonimbus. (c ) Photo d'un orage super-cellulaire, dans lequel la grêle peut se former. (d) Visualisation du courant ascendant incliné, puissant et durable, au sein du nuage super-cellulaire. Sur les schémas, les altitudes et températures approximatives sont indiquées.

\section{Démonstration avec le grêlon en pâte à modeler}

On explique maintenant plus concrètement comment se forme le grêlon dans l'orage supercellulaire, grâce à l'activité avec de la pâte à modeler. C'est d'abord l'adulte qui fabrique son grêlon devant tous les enfants, pour bien expliquer les mécanismes, en précisant que ce sera ensuite au tour des enfants de le fabriquer eux-mêmes.

Le support imprimé représente un orage super-cellulaire avec son ascendance rapide et durable (figure 3a). Dans les premiers kilomètres au-dessus du sol, la température de l'air est supérieure à $0^{\circ} \mathrm{C}$, la vapeur d'eau se condense sous forme de gouttelettes d'eau liquide, qu'on représente avec des billes de pâte à modeler bleue. On colle alors des billes bleues sur le support (figure 3b). Au dessus, la température est plus basse et il se forme des cristaux de glace, que l'on représente avec la pâte à modeler jaune. Ils sont majoritaires quand les températures sont inférieures à $-25^{\circ} \mathrm{C}$.

Certaines gouttelettes restent liquides à l'état surfondu jusqu'à $-25^{\circ} \mathrm{C}$ environ. La répartition de ces gouttelettes est très hétérogène, avec des zones où elles sont très concentrées et d'autres où elles le 
sont moins. Entre 0 et $-25^{\circ} \mathrm{C}$, on représente avec des billes bleues les gouttelettes dans les zones où elles sont très concentrées et avec des billes blanches les gouttelettes dans les zones où elles sont moins concentrées (figure 3b).

On précise bien qu'on ne colle les billes que sur la partie du support qui représente un nuage, puisque ce sont les gouttelettes d'eau et les cristaux de glace qui constituent le nuage. Il faut aussi faire attention à coller les billes de la bonne couleur dans la bonne zone de température. Pour éviter un moment de flottement, il est judicieux de préparer à l'avance une cinquantaine de billes de chaque couleur.

L'histoire du grêlon commence lorsqu'un petit cristal de glace pénètre dans le courant ascendant et est soulevé jusqu'à la zone de gouttelettes surfondues. On illustre cela en prélevant une bille de pâte à modeler blanche et en la plaçant au bas du courant ascendant. Soulevé par le flux ascendant, le petit grêlon rencontre des gouttelettes d'eau liquide surfondue qui gèlent en se collant à sa surface. Le grêlon se couvre alors d'une mince pellicule de glace. Les bulles d'air emprisonnées dans l'eau ont le temps de s'échapper avant la congélation. La glace, pure, est transparente. On la représente en bleu dans cette activité. Tout en expliquant ce processus, on fait se tamponner le grêlon en formation avec les billes bleues, et on recouvre sa surface de billes bleues en les aplatissant légèrement (figure 3c).

Au cours de son voyage dans le courant ascendant, le grêlon en formation traverse aussi des zones moins concentrées en gouttelettes. Il se refroidit, les gouttelettes rencontrées congèlent plus vite et l'air n'a pas le temps de s'échapper. Cette couche de glace est de couleur blanche. On la représente en blanc dans cette activité. Tout en expliquant ce processus, on recouvre la bille devenue bleue de petites billes blanches, jusqu'à ce que la bille soit entièrement devenue blanche (figure 3d).

Le grêlon traverse successivement des zones de fortes et de faibles concentrations en gouttelettes. On recouvre alors la grosse bille d'une nouvelle couche de billes bleues (figure 3e), puis blanches. Et ainsi de suite, plusieurs fois de suite. Un gros grêlon de $10 \mathrm{~cm}$ de diamètre peut ainsi contenir 5-6 couches successives. Lorsque le grêlon est suffisamment gros, et donc lourd, sa vitesse de chute (encadré 1) dépasse la vitesse de l'ascendance de l'air, et il tombe au sol (figure 3g).

On peut alors couper le grêlon ainsi obtenu en 2 avec un petit couteau en plastique. On observe alors des couches concentriques bleues et blanches (figure 3h), qui ressemblent à celles observées dans les vrais grêlons (figure 1d).

Figure 3. (a) Support cartonné et plastifié vierge. (b) Support plastifié quand toutes les billes de pâte à modeler sont en place. (c) Un cristal de glace tombé à la base de l'ascendance se recouvre d'une première couche de pâte à modeler bleue lors de son parcours dans le courant ascendant. (d) Il continue son parcours de la droite vers la gauche et se recouvre d'une première couche de pâte à modeler blanche en traversant des zones moins concentrées en gouttelettes. (e) Le grêlon se recouvre d'une nouvelle couche de pâte à modeler bleue en traversant une zone moins concentrée en gouttelettes. (f) Le grêlon continue encore son parcours vers la gauche et se recouvre d'une nouvelle couche de pâte à modeler blanche puis bleue. (g) Quand il est trop gros, le grêlon tombe. (h) Une fois terminé, le grêlon est coupé en deux, pour voir les couches concentriques. Ce grêlon a été fabriqué par l’un des enfants de l'école.

\section{Mise en ouvre de l'atelier avec les enfants}

C’est maintenant aux enfants de jouer ! Cet atelier a été testé dans une classe de maternelle avec des enfants de 4 à 6 ans (figure 4a). La discussion introductive a été faite avec la classe entière, puis l'activité s'est poursuivie par groupes de 8 enfants afin de mieux pouvoir les encadrer. Si le support est plastifié, un coup d'éponge humide suffit à le nettoyer pour qu'il soit réutilisé de nombreuses fois.

Chaque enfant a devant lui un support plastifié représentant l'orage super-cellulaire et une coupelle contenant un morceau de pâte à modeler blanche et un morceau de pâte à modeler bleue (figure 4a). Si le support est plastifié, les billes de pâte à modeler adhèrent mieux que sur du simple papier. On les accompagne pour qu'ils fabriquent les billes blanches et bleues, pour qu'ils les collent, puis pour 
qu'ils fabriquent le grêlon. A chaque étape, on rappelle le lien entre ce qu'ils font en pâte à modeler et le processus que ça représente en réalité. Une fois le grêlon terminé, chaque enfant le coupe en deux (figure 4b) pour constater les couches concentriques (exemple en figure 3f). Ils peuvent ensuite le rapporter à la maison pour le montrer fièrement à leurs parents.

Un accompagnement individuel est nécessaire, car les enfants diffèrent largement dans leur capacité de concentration, l'attention portée lors de la démonstration par l'adulte, leur habilité manuelle ou le temps qu'ils ont envie de consacrer à cette activité (figure 4c). Par exemple, dans la classe où l'atelier a été testé, certains enfants avaient fini au bout de 15 minutes, tandis que d'autres, plus appliqués et perfectionnistes, ont travaillé pendant plus de 30 minutes pour produire des grêlons de toute beauté (figure 3f). Il peut donc être utile de prévoir une activité pour les enfants ayant terminé plus rapidement.

Figure 4. Quelques photos de mise en œuvre dans une classe de maternelle : (a) les enfants travaillent par tables de 3 ; (b) un grêlon coupé en deux par l'un des enfants ; (c) huit exemples de grêlons fabriqués par des enfants. Photos prises par Chantal Lahanque. Notons que lors de cet essai en classe, le support était légèrement différent.

\section{Remerciements}

Nous remercions la directrice Chantal Lahanque et les enfants de l'école des Bambins de La Varenne où l'atelier a été testé avec des enfants pour la première fois. Nous remercions Michaël Kreitz et un relecteur anonymes pour leurs commentaires constructifs, ainsi que Brant Foote pour ses explications à la fois très détaillées et accessibles sur les mécanismes de formation des grêlons.

\section{Encadré 1 : taille des hydrométéores}

Les hydrométéores (gouttelettes d'eau dans les nuages, gouttes de pluie cristaux de glace, agrégats de cristaux, grésil, grêlons) sont classés selon leur taille. Les gouttelettes d'eau ont un diamètre compris entre 1 et $50 \mu \mathrm{m}$. Les gouttes de pluie mesurent entre $50 \mu \mathrm{m}$ et $5 \mathrm{~mm}$. La taille des cristaux de glace varie de 0.1 à $100 \mu \mathrm{m}$. Les agrégats de cristaux ou flocons de neige peuvent atteindre des dimensions supérieures à $1 \mathrm{~cm}$. Pour les particules givrées, plus compactes, on parle de grésil entre $100 \mu \mathrm{m}$ et $1 \mathrm{~cm}$ de diamètre. Au delà, il s'agit de grêle, les plus gros grêlons pouvant atteindre 10 $\mathrm{cm}$.

Les flocons formés de dizaines de cristaux enchevêtrés ont un diamètre pouvant aller jusqu'à 1 ou 2 $\mathrm{cm}$. Mais ce sont des objets complexes et fragiles. Ceci explique pourquoi sous les orages il ne neige pas : les cristaux de glace sont suffisamment petits pour fondre rapidement quand ils tombent du nuage. Quand aux flocons plus gros, ils se brisent en morceaux et fondent très vite lorsqu'ils rencontrent des températures supérieures à $0^{\circ} \mathrm{C}$. Dans le cas d'un orage printanier ou estival, seul le grésil et la grêle peut atteindre le sol sans fondre totalement.

\section{Encadré 2 : Vitesse de chute des hydrométéores}

La vitesse de chute d'un hydrométéore traduit un équilibre entre deux forces : le poids proportionnel à la masse, donc au volume, et le frottement de l'air qui dépend de la surface. Les hydrométéores tombent donc d'autant plus vite qu'ils sont denses et compacts (goutte de pluie, grêlon). A l'opposé, les agrégats complexes de cristaux formant les flocons de neige présentent une grande surface pour un faible poids et leur vitesse de chute est plus faible. Les hydrométéores tombent vers le sol lorsque leur vitesse de chute dépasse celle de l'ascendance de l'orage. Sinon, ils restent en suspension dans l'air et sont emportés avec le flux ascendant. 
En simplifiant le problème, on peut considérer que les gouttelettes d'eau et les petits cristaux de glace ont une vitesse de chute négligeable (ils suivent donc les mouvements de l'air) et que les flocons tombent avec une vitesse ne dépassant pas $4 \mathrm{~km} / \mathrm{h}$. C'est notre vitesse quand on marche. $\mathrm{La}$ vitesse de chute V (en m/s) du grésil ou de la grêle peut se calculer approximativement en fonction du diamètre $\mathrm{D}$ (en $\mathrm{m}$ ) par une relation du type $\mathrm{V}=488 \times \mathrm{D}^{0.84}$ (Heymsfield and Wright, 2014). Un grésil de $1 \mathrm{~mm}$ de diamètre tombe à une vitesse de $5 \mathrm{~km} / \mathrm{h}$, soit la vitesse d'une marche rapide, un grêlon de $5 \mathrm{~mm}$ de diamètre à $20 \mathrm{~km} / \mathrm{h}$, soit la vitesse d'un vélo, un grêlon de $2 \mathrm{~cm}$ à $65 \mathrm{~km} / \mathrm{h}$, soit un peu plus vite qu'une voiture en ville, un grêlon de $4 \mathrm{~cm}$ tombe à environ $120 \mathrm{~km} / \mathrm{h}$, soit comme une voiture sur l'autoroute, et enfin un grêlon exceptionnel de $10 \mathrm{~cm}$ à 250km/h, soit presque la vitesse d'un TGV!

On comprend donc que seules les ascendances les plus vigoureuses au sein des orages les plus intenses peuvent supporter les plus gros grêlons. Par exemple, un grêlon de $10 \mathrm{~cm}$ de diamètre ne peut se former que si l'ascendance atteint environ $250 \mathrm{~km} / \mathrm{h}$ !

\section{Encadré 3 : les orages super-cellulaires}

Les plus gros grêlons se forment dans les orages dits super-cellulaires (figure 1c). Ces orages ont une structure particulière qui les rend particulièrement puissants et durables.

En effet, dans les cumulonimbus isolés, l'évaporation partielle des gouttes de pluie lors de leur chute entraine des descentes d'air froid. Ces descentes alimentent une poche d'air froid qui s'étale près de la surface (figure 5a). L'air chaud qui alimentait l'ascendance est alors repoussé loin de l'orage. Privé du flux entrant d'air chaud et humide, le cumulonimbus se dissipe au bout d'une trentaine de minutes. Au contraire, dans un orage super-cellulaire, la variation de la direction et de l'intensité du vent environnant avec l'altitude confère à la masse nuageuse un large mouvement de rotation. Les courants ascendant chauds et humides et descendants plus froids sont organisés de façon non destructive. L'air froid déposé en surface facilite le soulèvement de l'air chaud qui produit l'eau nuageuse et précipitante dont l'évaporation dans l'air plus sec de moyenne troposphère donne naissance au courant descendant (figure 5b). L'orage super-cellulaire peut ainsi durer plusieurs heures. Il a le temps de devenir beaucoup plus puissant qu'un cumulonimbus isolé. Les orages super-cellulaires se développent dans des circonstances très particulières : une très forte instabilité convective avec de l'air très chaud et humide en basses couches et de l'air très sec en moyenne troposphère, une forte augmentation de la vitesse du vent et un changement de sa direction avec l'altitude. C'est la variation de la direction du vent avec l'altitude qui induit la rotation de l'orage. Ces conditions se retrouvent fréquemment au printemps ou en été dans les Grandes Plaines du centre des États-Unis, où les grêlons peuvent atteindre $10 \mathrm{~cm}$ de diamètre. Des super-orages de ce type se produisent aussi parfois en France. S'ils ne représentent que $10 \%$ environ des situations orageuses, on leur attribue la grande majorité des dévastations catastrophiques liées à la grêle.

Figure 5. Différence entre un cumulonimbus isolé (a) et un orage super-cellulaire (b). Les flèches rouges indiquent les ascendances, les flèches bleues indiquent les descentes d’air froid. Les cercles bordés de triangles bleus représentent les front des poches froides générés par les descentes d’air froid. Au dessus de l’orage super-cellulaire, la flèche noire indique le mouvement de rotation. A droite, les flèches indiquent la direction du vent dans l'environnement de l'orage super-cellulaire, avec l'exemple d'un profil typique dans les grandes plaines des Etats-Unis en été. Les nombres en marron indiquent la vitesse du vent en $\mathrm{m} / \mathrm{s}$.

Encadré 4 : l'eau surfondue et couches de glace concentriques dans le grêlon 
Les gouttelettes d'eau congèlent difficilement du fait de leur forte tension de surface. Aussi, il est possible d'observer des gouttes d'eau liquide, dites " surfondues » à des températures inférieures à $0^{\circ} \mathrm{C}$. En théorie, les plus petites gouttelettes de nuage (de diamètre inférieur à $10 \mu \mathrm{m}$ ) peuvent rester à l'état surfondu jusqu'à $-40^{\circ} \mathrm{C}$. En pratique, elles sont surtout fréquentes entre 0 et $-25^{\circ} \mathrm{C}$. Les gouttelettes congèlent lorsque la température devient très froide (environ $-10^{\circ} \mathrm{C}$ pour les plus grosses, $-40^{\circ} \mathrm{C}$ pour les plus petites), ou lorsqu'elles entrent en contact avec une autre particule (aérosol, cristal de glace).

Lorsque le grêlon rencontre des gouttelettes d'eau surfondue, l'eau surfondue recouvre plus ou moins totalement la particule de glace et remplit les interstices éventuels, puis elle congèle. Ceci a pour effet d'homogénéiser et de densifier la particule de glace initiale. Ce phénomène contribue à transformer des petits cristaux irréguliers peu denses en grésil et grêlons plus sphériques et plus denses.

La congélation de l'eau liquide surfondue libère de la chaleur latente qui réchauffe le grêlon. Ainsi, plus le grêlon agglomère rapidement des gouttelettes d'eau, plus il se réchauffe. La répartition des gouttelettes surfondues est très hétérogène dans l'ascendance. Quand le grêlon en formation arrive dans une zone très concentrée en gouttelettes surfondues, la chaleur latente libérée lors de la fonte réchauffe le grêlon. L'eau congèle alors plus lentement et les bulles d'air s'échappent facilement de la glace lors de la congélation, formant une couche de glace transparent. Inversement, quand le grêlon en formation arrive dans une zone moins concentrée en gouttelettes surfondues, il y a moins de chaleur latente dégagée et le grêlon se refroidit. L'eau congèle plus rapidement et les bulles d'air restent emprisonnées dans la glace froide, formant une couche de glace blanche. Ainsi, les couches concentriques du grêlon s’explique par la traversées de zones plus ou moins concentrées en gouttelettes, au cours de son long (une dizaine de minutes) parcours dans le courant ascendant entre 0 et $-25^{\circ} \mathrm{C}$.

Jusqu'aux années 1980s, on supposait que les couches concentriques des grêlons s'expliquaient par de multiples montés et descentes dans le courant ascendant. L'émergence des données radar Doppler permettant de mesurer la vitesse et la direction des vents (Tabary, 2013) ont permis de calculer des trajectoires réalistes de grêlons. On s'est alors rendu compte que la plupart des grêlons grossissaient lors d'un parcours unique au travers du courant ascendant, à une altitude quasiconstante (Foote, 1984 ; Miller et al, 1988). Ce sont les variations horizontales des conditions de croissance, plus que les variations verticales, qui expliquent les couches concentriques.

\section{Références}

Foote, G. B. (1984). A study of hail growth utilizing observed storm conditions. Journal of climate and applied meteorology, 23(1), 84-101.

Heymsfield, A and Wright, R.L. (2014) Graupel and Hail Terminal Velocities: Does a “Supercritical” Reynolds Number Apply? J. Atmos. Sci., 71, 3392-3403

Miller, L. J., Tuttle, J. D., \& Knight, C. A. (1988). Airflow and hail growth in a severe northern High Plains supercell. Journal of the atmospheric sciences, 45(4), 736-762.

C. Risi, J-L Dufresne, J-Y Grandpeix, S Labetoulle, G Sèze, A Spiga (2014) Comment se forment les nuages ? L'expérience du nuage dans une bouteille. La Météorologie, 86 : 20-22.

C. Risi, V Journé, J-L Dufresne, J-Y Grandpeix, A Spiga (2016). Mise en évidence de la chaleur latente liée à l'évaporation et à la condensation de l'eau: Applications au fonctionnement des orages. La Météorologie, 94 : 15-18 
F. Roux (1991). Les orages. Météorologie des grains, de la grêle et des éclairs. Payot, Paris, 358 p.

Tabary, P, Augros, C, Champeaux, J-L, Chèze, J-L, Faure, D, Idziorek, D, Lorandel, R, Urban, B, Vogt, V (2013). Le réseau et les produits radars de Météo-France. La Météorologie, 83:15-27. 
a

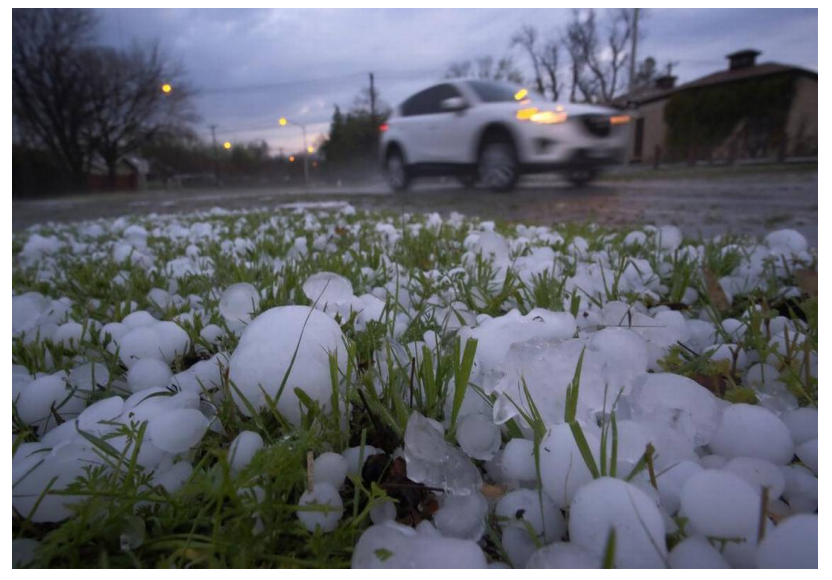

c

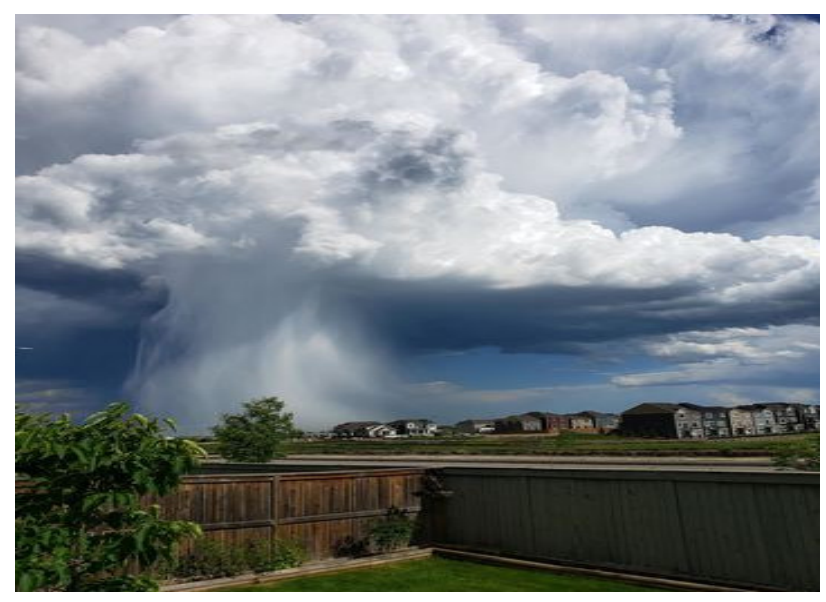

oe Mendoza, Silverado Plains Close, Calgary
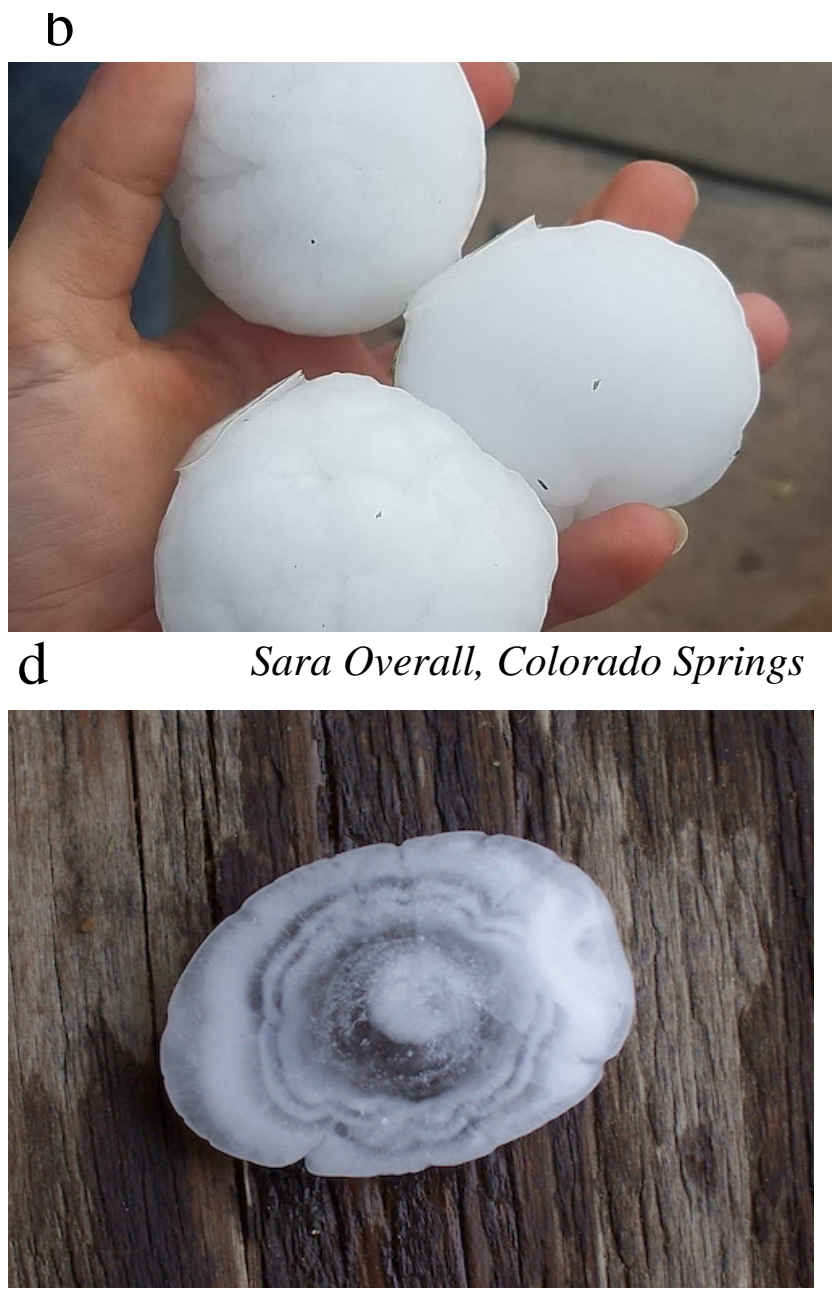

Licensed under the GFDL by the author

Figure 1: 


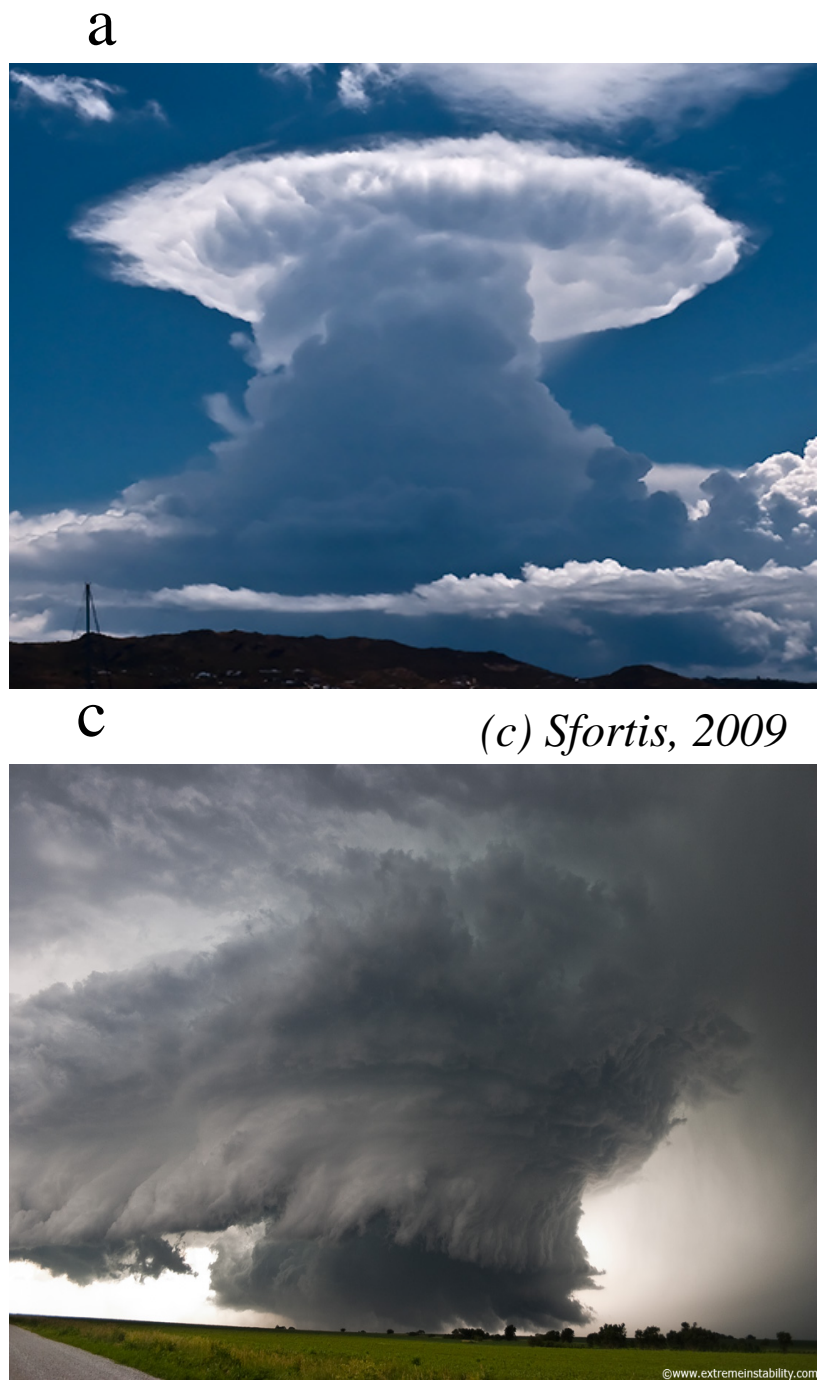

D
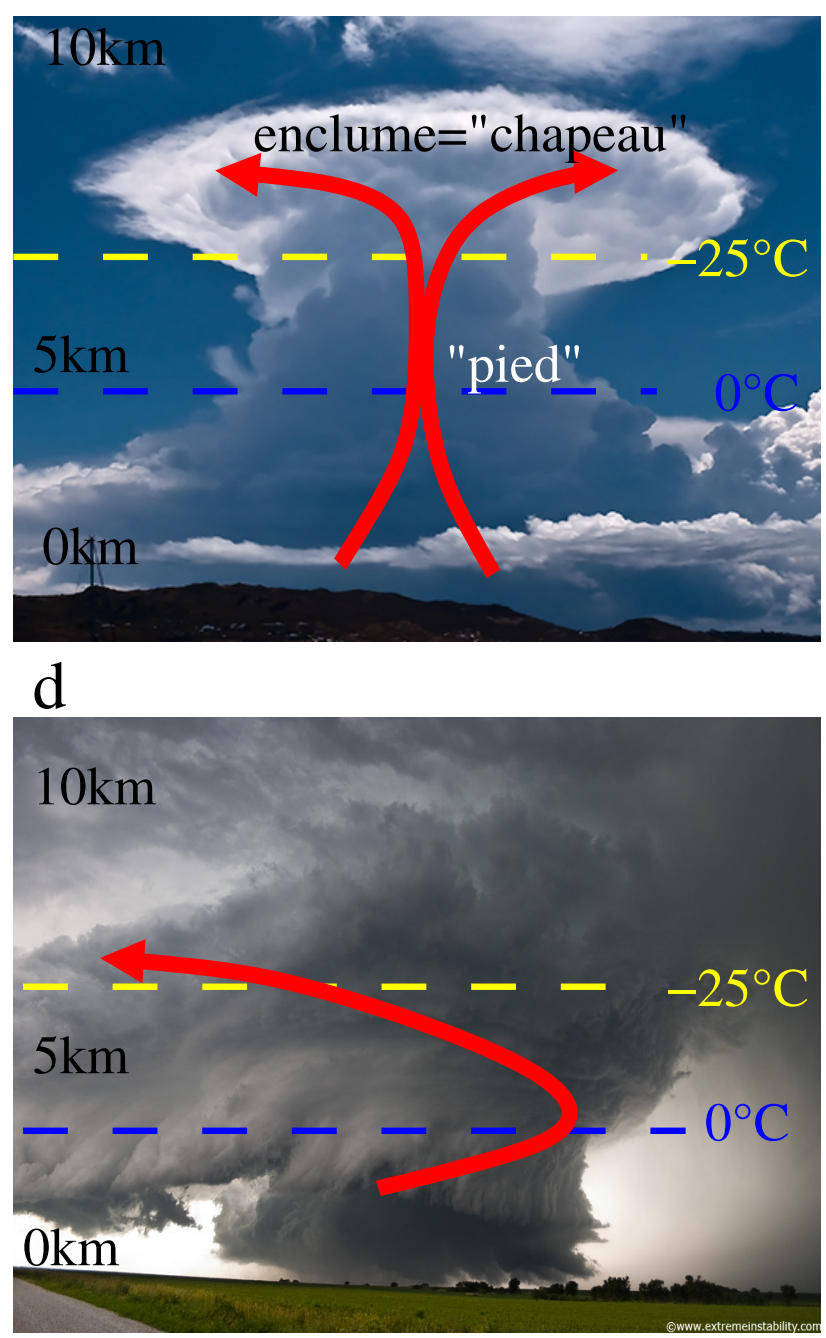

(c) www.extremeinstability.com

Figure 2: 
a

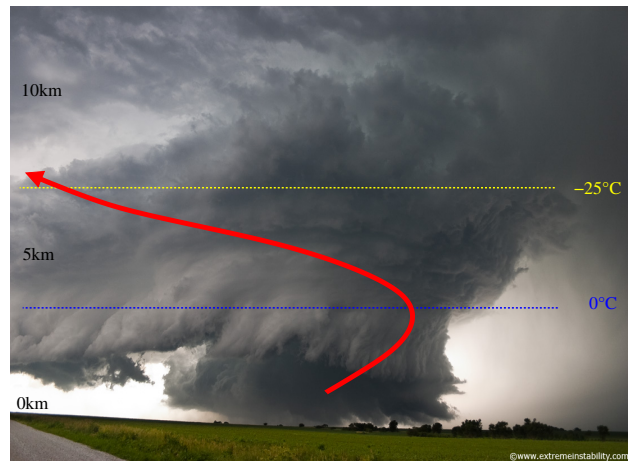

c

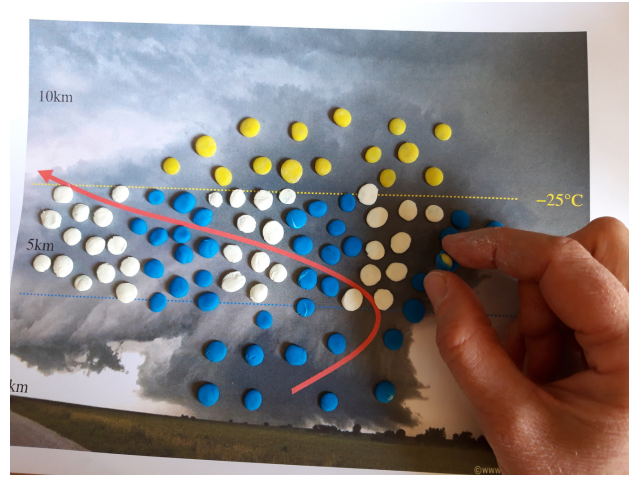

e

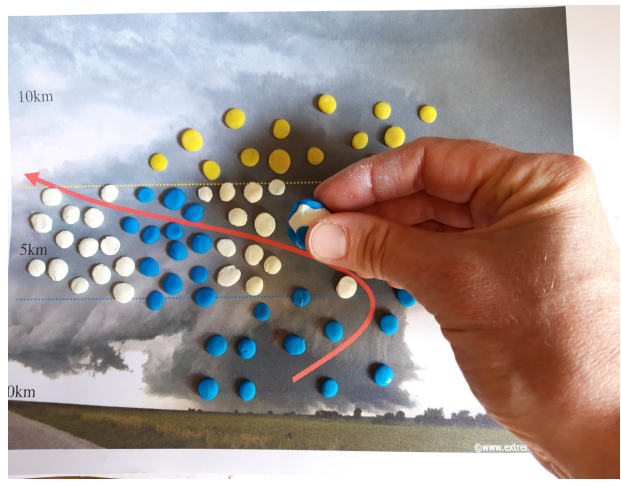

g

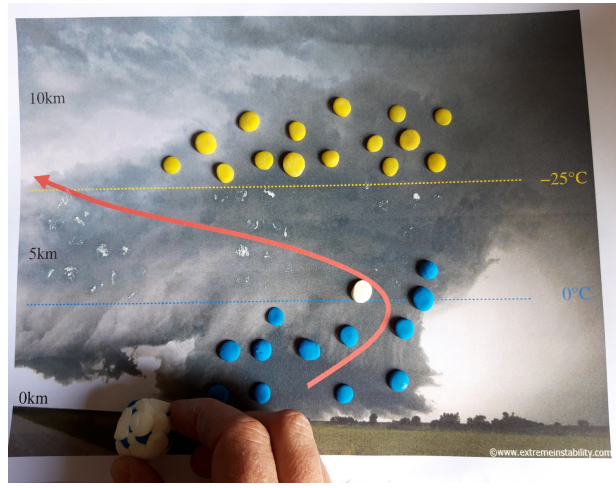

b

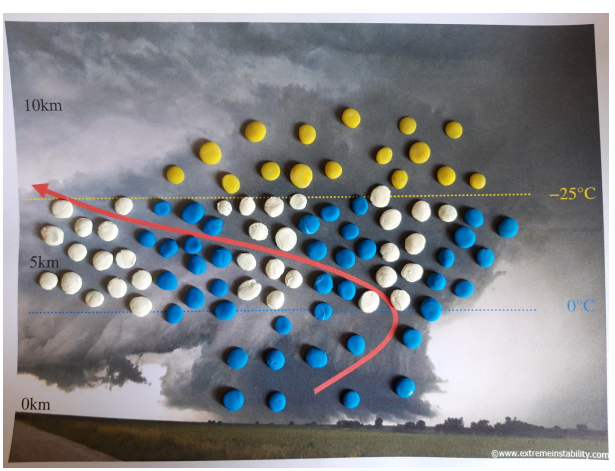

d
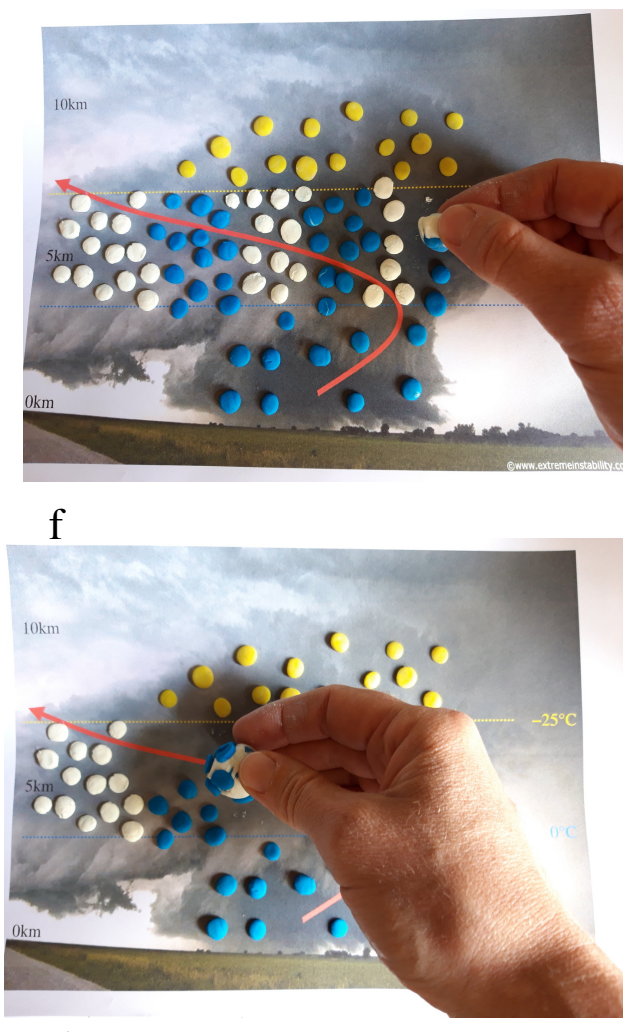

h

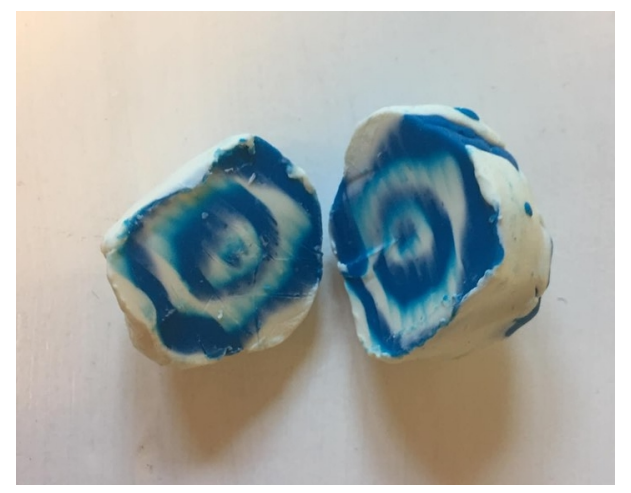

Figure 3: 
a

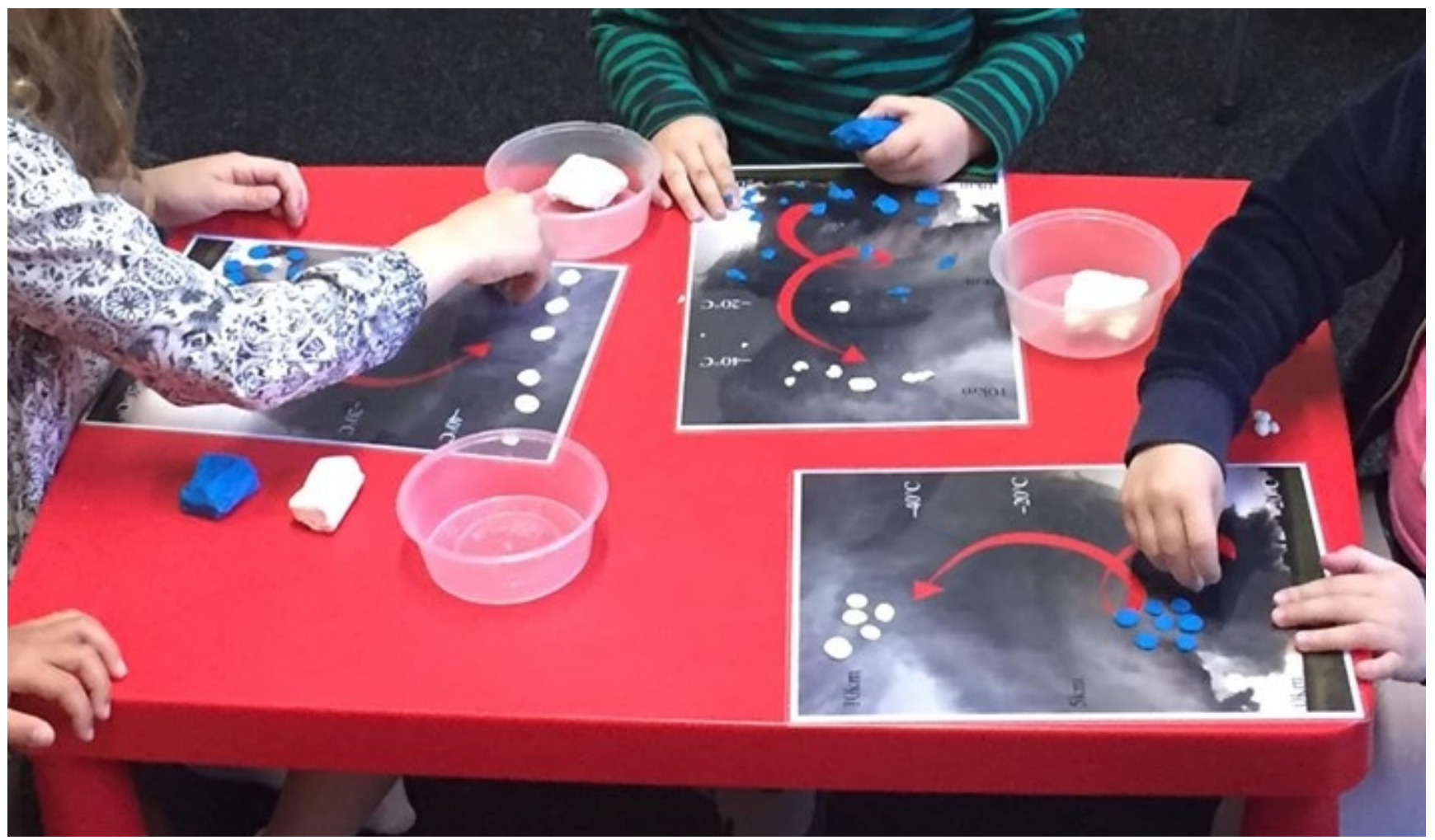

b

C
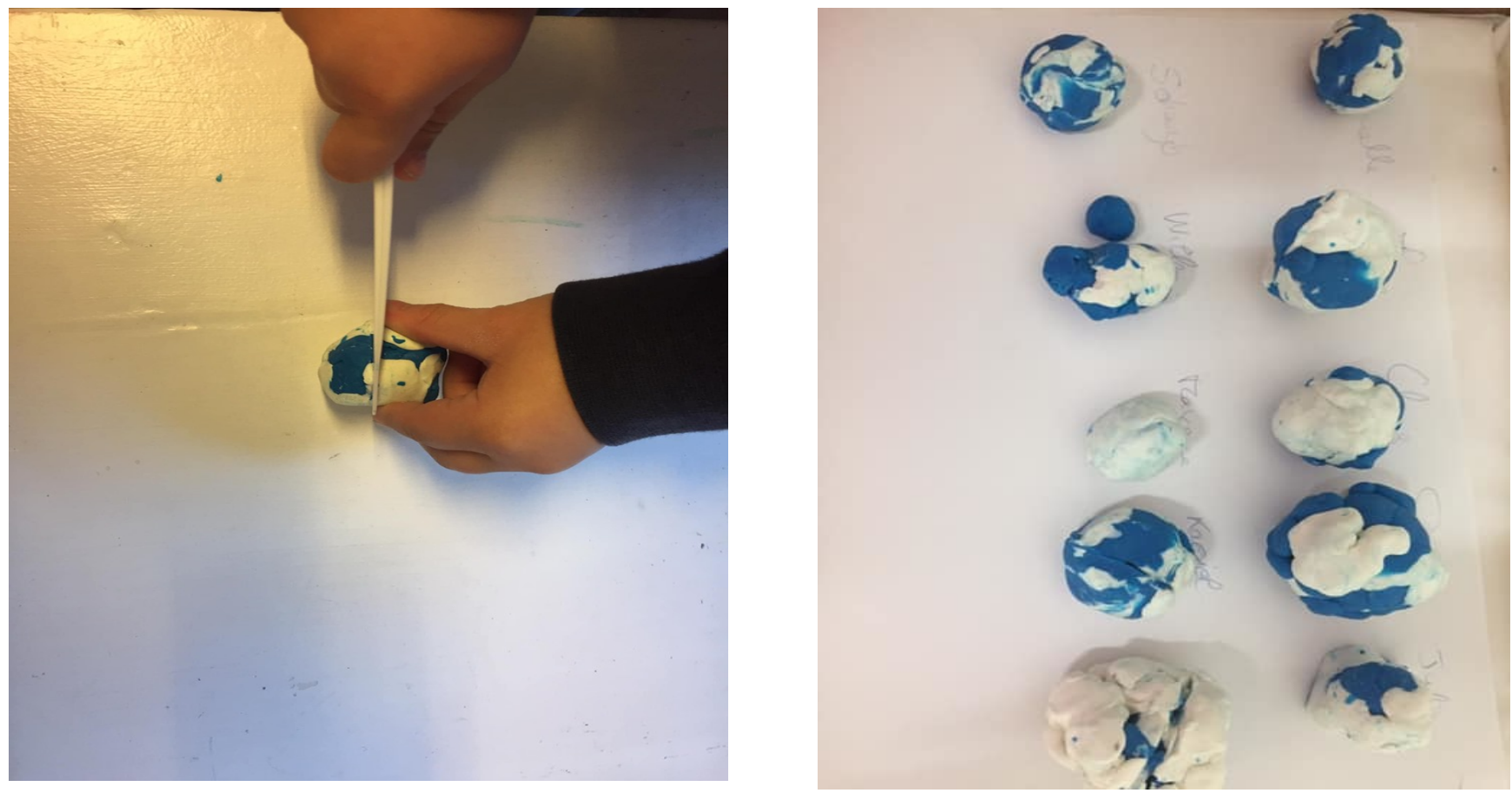

Figure 4: 
(a) Cumulonimbus isolé

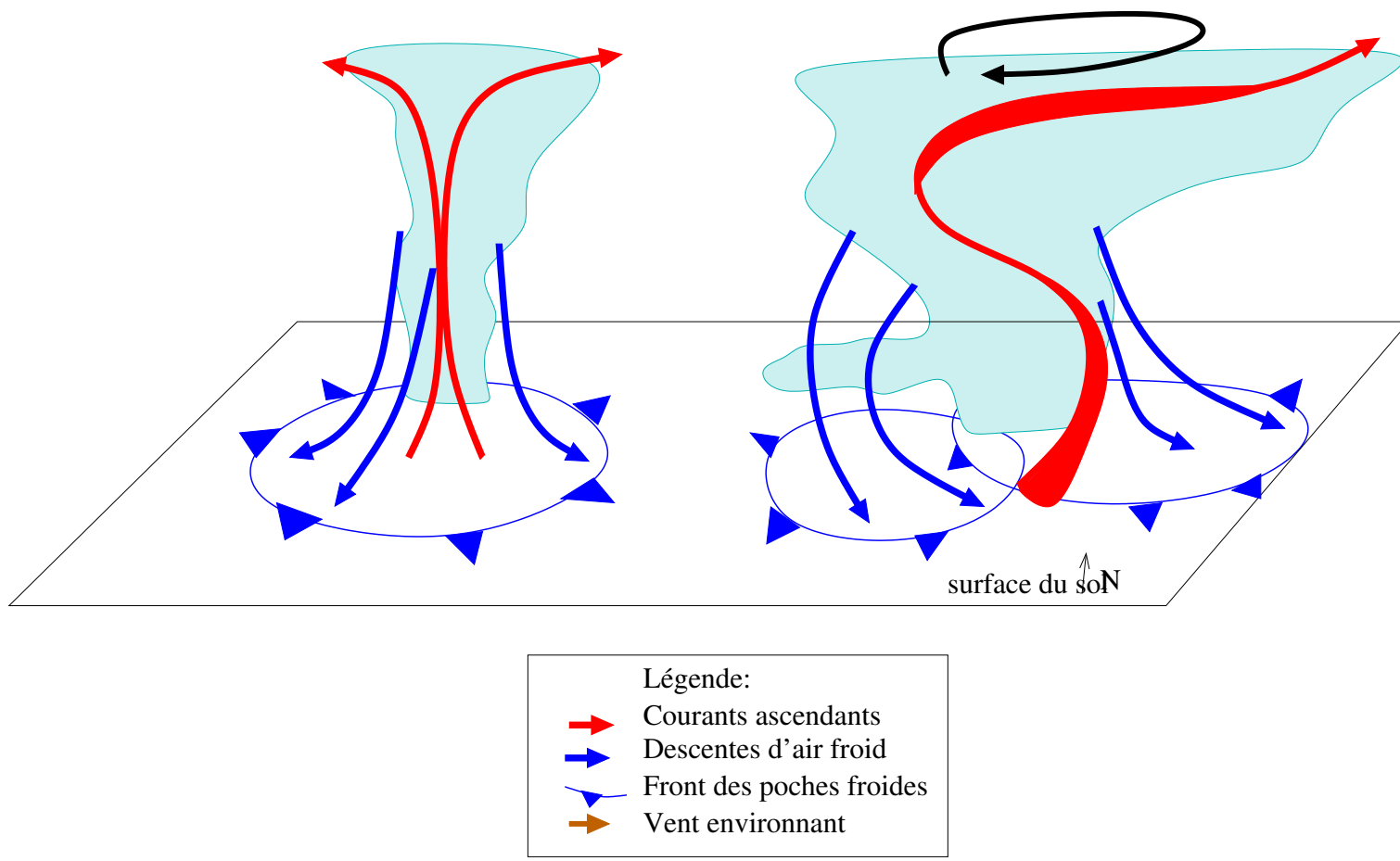

altitude $(\mathrm{km})$

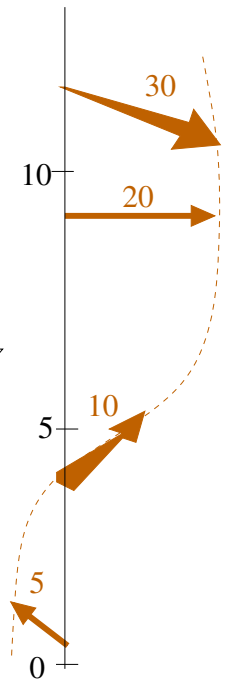

Figure 5: 